\title{
Una lectura de la doctrina kantiana del genio
}

\author{
Luciana Martínez ${ }^{1}$
}

Resumen: En este artículo se examina la doctrina kantiana del genio. En primer lugar, se estudia el desarrollo de esa doctrina en el período precrítico. Se muestra que durante ese período Kant restringe el ámbito de injerencia del genio. Hacia comienzos de los ' 80 , el genio es el productor de bellas artes. En segundo lugar, se estudia la argumentación crítica. Se detalla la naturaleza del genio y se explica su función sistemática para la Crítica del Juicio.

Palabras clave: Kant - genio - arte - período precrítico - Crítica del Juicio

\section{An Interpretation of Kantian Doctrine of Genius}

Abstract: In this paper, the Kantian doctrine of genius is examined. Firstly, the development of this doctrine during the pre-critical period is studied. It is shown that Kant did restrict the genius' scope in this period. In the early eighties, genius was already considered only as the maker of fine arts. Secondly, his critical argumentation is studied. The features of genius and its systematic function within the Critique of Judgment are then explained.

Keywords: Kant - genius - fine arts - pre critical period - Crítique of Judgment

\footnotetext{
${ }^{1}$ Luciana Martínez es doctora en Filosofía por la Universidad de Buenos Aires. Ha realizado una investigación posdoctoral financiada por el Consejo Nacional de Investigaciones Científicas y Técnicas (CONICET) y actualmente se desempeña como investigadora en la Universidad Kant de Kaliningrado (IKBFU). Participa en numerosos grupos y equipos de investigación y ha publicado varios artículos sobre la filosofía teórica de Kant.
} 


\section{Introducción}

En este texto se examina la doctrina kantiana del genio, con el propósito de exhibir los motivos de su necesaria, y exclusiva, inscripción en el dominio de la producción artística. Intentaré mostrar que el desarrollo de la visión crítica de la belleza conduce al filósofo a redefinir el ámbito de incumbencia del genio. Para ello, en primer lugar examinaré la evolución del concepto en el período precrítico de la producción del filósofo, con el fin de mostrar que ya en los '70 Kant pensaba que el genio tenía un ámbito de intervención restringido que, no obstante, alcanzaba todavía a algunas ciencias. Hacia 1780, de acuerdo con los documentos disponibles, ese ámbito se restringe aún más, reduciéndose apenas al ámbito de la producción artística. En segundo término, se exhibirán algunos aspectos de la doctrina del genio, tal y como se presenta en la Crítica del Juicio. El objetivo general de este examen es mostrar que el genio tiene una función sistemática específica en este texto y que una definición específica del término es necesaria para que el genio satisfaga esa función.

En un artículo publicado en 2001, C. Wenzel ya ha investigado las razones que llevaron a Kant a abandonar la noción de la genialidad matemática ${ }^{2}$ y a restringir la doctrina del genio al ámbito de la producción de bellas artes. El tratamiento del tema por parte de Wenzel tiene dos diferencias significativas con el tratamiento que se desarrolla en el artículo presente. Por un lado, Wenzel considera que la restricción de la Crítica del Juicio constituye una exclusión de las prácticas matemáticas del ámbito de injerencia del genio. En este artículo sostengo que esa restricción había sido realizada mucho antes y que lo excluído por Kant en 1790 es la incidencia del genio en el ámbito de otras ciencias, tales como la Filosofía.

En segundo lugar, Wenzel vincula el cambio principalmente a dos aspectos de la doctrina. Por un lado, el genio se asocia con los productos de la imaginación. En este artículo intentaremos mostrar que en efecto esa asociación está relacionada de manera histórica con la restricción del genio al ámbito del arte; en particular, es la lectura de y la discusión con Gerard lo que desencadena el cambio en el joven Kant, a mediados de la década de 1770. Por otra parte, para Wenzel Kant vincula la confusión por la que se habla de genio más allá del arte con una falta de precisión conceptual: se confunde el genio con el talento. En contra de esta tesis, sin embargo, podemos ver que en los años '70 Kant distingue cuidadosamente el genio entre los talentos, y adscribe genialidad a ciertos sectores de la producción de conocimiento. En la Crítica del Juicio, por su parte, señala de manera explícita que el genio es un talento, es decir, un don natural. ${ }^{3}$

Este artículo, como hemos adelantado, tiene dos partes. En la primera examinaremos la evolución de la doctrina del genio durante la década de 1770, con el fin de exhibir el cambio que se produce, especialmente desde mediados de esa década y en estrecha relación con la lectura de Gerard. En la segunda parte, mostraremos que una noción específica de genio es requerida por la estructura sistemática de la argumentación de la Crítica del Juicio.

\footnotetext{
${ }^{2}$ Esta investigación, como el mismo Wenzel señala, tiene antecedentes en TONELLI, "Kant's Early Theory of Genius" y GIORDANETTI, "Das Verhältnis von genius, Künstler und Wissenschaftler in der Kantischen Philosophie".

${ }^{3}$ Las obras de Kant se citan de acuerdo con las convenciones estándar, especificadas en la revista Kant Studien. KU, §46, AA 5: 307.
} 


\section{La evolución de la doctrina del genio ${ }^{4}$}

La Crítica del Juicio se publicó en 1790. Tenemos testimonios dos décadas anteriores del interés de Kant por el concepto de genio. En su mayor parte, estos testimonios son anotaciones de los estudiantes en sus clases de Antropología. En los '70 Kant explicaba y describía este concepto en sus clases. Curiosamente, de acuerdo con los testimonios disponibles, Kant se alejaba de los textos que debía explicar en clase. La Metafísica de Baumgarten y la Lógica de Meier ni siquiera incluían este concepto en el que Kant, sin embargo, sí se demoraba en sus cursos de Antropología y Lógica.

Una de las numerosas disciplinas que Kant enseñaba en las aulas se llamaba "Enciclopedia Filosófica". Este curso consistía en una suerte de ordenado, esquemático, resumen introductorio a los conocimientos de la época. Para dar estas clases, Kant empleaba un libro escasamente considerado por los estudiosos de su filosofía. Su autor, Feder, sí usaba la palabra "genio". Y la usaba en un contexto muy específico: para investigar cuál es la naturaleza anímica de un buen filósofo. Feder indagaba por el genio filosófico, es decir las aptitudes o disposiciones del hombre que hace filosofía. La razón de esta indagación estaba vinculada con la necesidad de diferenciar los procedimientos para conocer en matemática y los procedimientos para conocer en filosofía. ${ }^{5}$

Es interesante que durante toda la década de 1770, de acuerdo con los testimonios que tenemos, Kant consideró que era necesario genio para filosofar. Es decir, era necesario un espíritu libre de reglas, un espíritu que no imitara, una disposición que no podía aprenderse, ni enseñarse, ni reproducirse para ser filósofo o artista. La matemática, en cambio, se presentaba para Kant, durante todo este período, como una ciencia de reglas. Las ciencias de reglas no requieren genio, que, en cambio, se vincula con una producción autónoma y original.

En las lecciones de lógica de la misma época, por ejemplo, Kant distinguía los conocimientos que podemos adquirir por aprendizaje y el conocimiento que obtenemos por el mero sano entendimiento ${ }^{6}$. En este sentido, cabe diferenciar aquellos conocimientos que pueden ser enseñados y los que no ${ }^{7}$. Estos últimos pertenecen a la incumbencia del genio. Este mismo tipo de consideraciones se encuentra en la base de la diferenciación entre la matemática y la filosofía. Los argumentos con los que Kant establecía el límite entre ambas ciencias son, en verdad, numerosos. Uno de ellos involucra la doctrina del genio. De la misma manera, en las lecciones de antropología de comienzos de la década, la matemática se describe como un arte que puede enseñarse. ${ }^{8}$ En ella hay reglas ${ }^{9}$ que guían los procedimientos y esas reglas pueden dictarse ${ }^{10}$. El caso de la filosofía es diferente. En ella no hay contenidos para

\footnotetext{
${ }^{4}$ En este apartado se recogen los resultados de algunos trabajos previos. En ellos examino los detalles de la evolución de la doctrina del genio en el período precrítico. Cf. MARTÍNEZ, "El desarrollo del genio artístico". 5 FEDER, Grundriß, 『4. Cf. también MARTINEZ, "Die Lehre vom Genie in Kants Vorlesungen über Philosophische Enzyklopädie".

${ }^{6} \mathrm{~V}$-Log/ Blom, AA 24:165.

${ }^{7} \mathrm{~V}-\mathrm{Log} / \mathrm{Phil}, \mathrm{AA} 24: 321 \mathrm{~s}$.

${ }^{8}$ V-Ant/Col, AA 25:164; V-Ant/Par, AA 25: 364, V-Ant/Fried, AA 25: 556.

${ }^{9}$ PhilEnz, AA 29:5.

${ }^{10}$ En este sentido, en contra de los que sostiene C. Wenzel, es inusual (aunque no inexistente) la atribución de genio al desarrollo de la investigación matemática, incluso a comienzos de la década de 1770. El artículo de Wenzel, sin embargo, constituye una explicación interesante de los motivos por los que para Kant no puede
} 
enseñar. Para Kant, no se ha escrito todavía un compendio de la filosofía. Además, la filosofía debe desarrollar un método y no hay reglas para la elaboración del método ${ }^{11}$.

Durante los primeros años de la década, el genio se describía como un espíritu original, contrapuesto al espíritu de la imitación ${ }^{12}$. Quisiera demorarme en la noción de espíritu porque va a ser importante también en el criticismo. El concepto de espíritu tenía una extensión diferente, más amplia, de la del concepto de genio. Por una parte, también la imitación involucra espíritu. Por otro lado, el espíritu se presenta como un principio vital, como algo que vivifica aquello en lo que se encuentra. ${ }^{13}$ El genio, por su parte, es innato, raro e inusual. ${ }^{14}$ Es un espíritu original, que crea y lo hace sin instrucciones ni reglas. ${ }^{15}$

Hay relativo consenso en el ámbito de las investigaciones kantianas respecto de la tesis según la cual la lectura y la discusión del texto An Essay on Genius, de Alexander Gerard fue crucial para Kant. El texto de Gerard se publicó, en inglés, en 1774. Dos años después, en 1776, se publicó su traducción al alemán, a cargo de Christian Garve. ${ }^{16}$ En este texto, Gerard presenta una doctrina del genio como inventor. La creación involucra todas las facultades del hombre. La principal de ellas es, sin embargo, la imaginación. Gerard desarrolla una cuidadosa argumentación para justificar la tesis según la cual esta facultad es la más importante para la producción genial.

Un elemento de esa argumentación consiste en la diferenciación entre la invención y el aprendizaje. Para Gerard, la capacidad de aprender, a diferencia de la capacidad de inventar, es común a todos los hombres. Alguien que no tenga esa capacidad será un monstruo. Por otro lado, el aprendizaje puede ser explicado a partir de las otras facultades del hombre, sin tomar en consideración la imaginación, que es propia del genio. Gerard considera que la actividad genial se expresa tanto en la ciencia como en el arte. En la ciencia, se necesita genio para crear conocimiento verdadero. En el arte, se necesita genio para crear belleza. En ambos casos, la facultad rectora en la invención es la imaginación. ${ }^{17}$

Entre 1776 y 1778, Kant habría escrito una anotación acerca del texto de Gerard ${ }^{18}$. Esta anotación es el comentario más detallado de Kant sobre este texto. ${ }^{19}$ En ella, el filósofo de Königsberg critica la concepción del genio como facultad, que atribuye a Gerard. Como hemos indicado antes, empero, Gerard no identifica al genio con una capacidad, sino que sostiene que el genio tiene todas las facultades. Sin embargo, la facultad característica del

haber genio (ni gusto) en el ámbito de la matemática. Cf. WENZEL, "Beauty, Genius, and Mathematics: Why Did Kant Change His Mind?”.

11 V-Anth/Fried, AA 25: 556.

${ }^{12}$ V-Log/Phil, AA 24: 493; V-Log/ Blom, AA 24: 299. Es importante reparar en esta distinción temprana entre ciencias de genio y ciencias de la imitación y el aprendizaje. Para Giordanetti, esta distinción es una consecuencia de la recepción de Gerard. Cf. GIORDANETTI, "Kant e Gerard. Nota sulle fonti storiche della teoria kantiana del 'genio", 688 ss. Sin embargo, estas fuentes indican que Kant había desarrollado la diferenciación antes de tener acceso al texto de Gerard.

13 V-Ant/ Par, AA 25: 437; V-Ant/Col, AA 25: 167; V-Ant/Fried, AA 25: 557.

14 V-Ant/ Fried, AA 25: 556s.

15 Met L1, PM 164.

16 Puede encontrarse un comentario sobre la publicación y la recepción de este texto de Gerard en KLUKOFF, "Alexander Gerard. An Essay on Genius (1774), edited by Bernhard Fabian (Theorie und Geschichte der Literatur und der Schönen Künste, vol. III). München. Wilhelm Fink Verlag. 1966. vii + 459 pp. DM.52”.

${ }^{17}$ Este párrafo es un resumen de GERARD, An Essay on Genius., parte 1, secciones 1 y 2.

18 R949, AA 15: 420s.

${ }^{19}$ Joãosinho BECKENKAMP ( "Kant und Gerard über Einbildungskraft" "Kant e Gerard sobre imaginação" ) sostiene que las críticas de Kant no se basan en el texto de Gerard, sino en un comentario de este texto, escrito por Johann N. Tetens.

: : : Cadernos de Ética e Filosofia Política | Número 38 (1) | 1º semestre de 2021 | Página 88 : : : 
genio es, para él, la imaginación. Para Kant, según la fuente mencionada, el genio no se identifica con una facultad, sino con la vivificación de nuestras facultades por medio de una idea. El concepto de la vivificación y la noción de una idea no son nuevos en las fuentes kantianas, pero sí lo es esta manera de caracterizar al genio. Por otro lado, en la reflexión el filósofo concede la consideración del genio como necesario para inventar.

En las lecciones de esta época se desarrolla una doctrina del genio que mantiene algunos elementos de las lecciones previas, pero también incorpora algunas novedades. Kant señala la diferencia entre descubrir e inventar. El descubrimiento se refiere a una cosa que ya existía. El ejemplo elegido por Kant es el descubrimiento de América. El objeto ya existente pero desconocido se trae a la luz con el descubrimiento, que no produce nada nuevo. Inventar, en cambio, involucra crear algo que antes no estaba. El ejemplo kantiano es el teorema de Pitágoras. ${ }^{20}$

Otra novedad que también está vinculada con el debate con Gerard es la indagación acerca del principio de la originalidad del genio. Para Kant, el carácter original, que no imita ni sigue reglas, sigue siendo el rasgo distintivo del genio. Pero para él ese principio no es la facultad de la imaginación, ya que los productos de esta facultad son, por sí mismos, caóticos. Lo que caracteriza al genio no es la imaginación, sino el espiritu. El espíritu, sin embargo, no es una facultad. Antes bien, es un principio que proporciona unidad y armonía a las facultades. ${ }^{21}$ En el concepto de espíritu así definido y en la consideración de los productos de la imaginación como no ordenados se cifra una visión crítica del planteo de Gerard.

Kant todavía sostiene la clasificación entre las ciencias que pueden aprenderse, como la matemática, y las ciencias de genio, como la filosofía. En este sentido, la filosofía tiene un origen común con el del arte. Poco después, a comienzos de la década de 1780, este rasgo de la filosofía se cancela. El genio se presenta en estas anotaciones asociado a la creación poética y caracterizado por la imaginación creativa. ${ }^{22}$ Así, positivamente la doctrina del genio se restringe, de manera explícita, al ámbito de la producción de arte.

La contracara de esto es que en el tratamiento de la diferencia entre la filosofía y la matemática se omite la noción de genio. Kant menciona diferentes tipos de "cabeza" $(\text { Kopf })^{23}$ y señala que la filosofía requiere ingenio. ${ }^{24}$ Ser un genio ${ }^{25}$, en cambio, significa tener una mente original. El genio requiere un talento que no puede sustituirse por el esfuerzo ${ }^{26}$. Los productos de la imaginación parecen ser un resultado de este tipo de talento. Por esta razón,

\footnotetext{
${ }^{20} \mathrm{~V}$-Ant/Pil, AA 25: 758.

21 V-Ant/Pil, AA 25: 782.

$22 \mathrm{~V}$-Ant/Mensch, AA 25: 991.

${ }^{23}$ Esta noción aparece en las lecciones previas y se encuentra ya en la versión alemana de la Metafísica de Baumgarten. El término, en este texto de Baumgarten, hace referencia a una peculiar disposición de nuestras facultades, que nos torna más apto en uno u otro ámbito del saber (matemático, histórico, filosófico, poético, mecánico). Cf. Baum, Met. \476. En la traducción de Meier, BAUMGARTEN, Metaphysik. Ins Deutsche übersetz̨t von Georg Friedrich Meier, 148.

24 V-Ant/Mensch, AA 25: 1054.

${ }^{25}$ Es importante señalar que en estas anotaciones se distingue entre "ser genio" y "tener genio". Cf. V-Anth/ Mensch, AA 25: 1061. Algunos autores, como Bruno, consideran que esta distinción aparece y se torna significativa después de Kant. Ortland muestra que ya había sido desarrollada en el siglo XVII.Véase ORTLAND, "Genius", 675.

${ }^{26}$ Esta representación del genio y su relación con el empeño ya había sido discutida en la literatura inglesa, desde que E. Young afirmó que el genio es un don y que no requiere ningún esfuerzo. Véase YOUNG, Conjectures on Original Composition: 6.
} 
no se encuentra genio en la ciencia, sino sólo en la poesía. Genios son los poetas y los artistas, no los científicos y filósofos. ${ }^{27}$

La objeción a la posición de Gerard es, una vez más, explícita. La imaginación es necesaria para el genio, pero no lo define. Y no hay genio en filosofía y en ciencia. ${ }^{28} \mathrm{El}$ concepto de genio se define en estas clases como un "talento original" y no como un talento prominente o más desarrollado. ${ }^{29} \mathrm{El}$ término proviene del latín genius ${ }^{30}$ y significa un espíritu peculiar que acompaña a las personas desde su nacimiento ${ }^{31}$. Genius proviene de gignere ${ }^{32}$ y permite resaltar el carácter original. La originalidad del genio se opone al espíritu de imitación, a todo aquello que puede enseñarse y a la producción que sigue reglas y modelos ${ }^{33}$.

A comienzos de la década de 1780, un elemento novedoso de la presentación que se vincula con este rasgo de la genialidad es la mención de Shakespeare. El autor inglés era un tema de discusión en la estética de la época en virtud de su deliberada infracción de las leyes del teatro clásico. En particular, Kant lo mencionaba por la omisión de la regla de la unidad del espacio en su obra. Kant, al igual que Gerard, presentaba a Shakespeare como a un genio $^{34}$. Su imaginación no podía estar restringida por reglas. Shakespeare se presenta como un maestro de las reglas y no como el esclavo de ellas. Él no se encuentra compelido a subsumir su obra a reglas arbitrarias. Ahora bien, esta peculiar relación del artista con las reglas no es universalizable, no vale para todos ni para todos los ámbitos de la vida de esos hombres singulares. ${ }^{35}$ En cambio, esa ruptura con la regla se presenta como una licencia. Fuera del ámbito de ella, infringir las reglas es un error. La genialidad compensa este error, pero el gesto no debe ser reproducido ${ }^{36}$.

A lo largo de la década de 1780, hasta la publicación de la Crítica del Juicio, las tesis principales de Kant acerca del genio se mantienen, de acuerdo con los testimonios disponibles. ${ }^{37}$ Todos los hombres tenemos dones naturales, que nos tornan talentosos en diversos ámbitos de la experiencia. El genio es un tipo de don natural que se tiene o no se tiene, que se debe cultivar pero no puede generarse por instrucción. Por esta razón, las ciencias, para las que sí podemos hacernos aptos por instrucción, no son producto de genio. El genio, dice Kant, es creador de arte.

\footnotetext{
27 Debemos mencionar en este punto que en estos mismos años Kant utiliza la noción de genio para hacer referencia a los grandes griegos que originaron la lógica (V-Met/Volck, 28: 368), así como a los requisitos necesarios para desarrollar una filosofía buena y comprensible (V-Log/Wien, AA 24:796).

${ }^{28} \mathrm{~V}$-Ant/Mensch, AA 25: 1055.

${ }^{29} \mathrm{~V}$-Ant/Mensch, AA 25: 1056.

${ }^{30}$ Fricke encuentra una diferencia entre el significado del término alemán (Genie) y el del término latino (genius) para Kant. Cf. FRICKE, "Genius", 758.

${ }^{31}$ Este comentario de Kant se identifica con las definiciones clásicas del término latino. Cf. Valpy 1828, 174.

${ }^{32}$ En de Vaan, el verbo “gigno, -ere” se define como 'crear, engendrar' (2008:260). En Ernout et Meillet se presenta un significado derivado nominal que involucra "una divinidad generatriz que preside el nacimiento de cada uno" (2001: 270 s.)

33 V-Anth/ Mensch, AA 25: 1056.

${ }^{34}$ La figura de Shakespeare y su ruptura con las reglas del teatro clásico eran objeto de gran discusión, como hemos señalado. En Alemania, muy cercano a Kant, Herder había desarrollado una frenética defensa del autor británico. Cf. HERDER, "Shakespeare".

35 Quizás en relación con este punto sea conveniente pensar la noción del “terror del genio” de DESMOND ("Kant and the Terror of Genius: Between Enlightenment and Romanticism"). Este autor hace referencia al hecho de que el genio no encaja en la descripción de las facultades regulares y las formas de creación estándar. Por esta razón, para el autor Kant se inscribe, en su doctrina del genio, entre la ilustración y el romanticismo. 36 V-Anth/ Mensch, AA 25: 1057.

${ }^{37}$ Cf. V-Anth/ Mrongovius (83/84), V-Anth/Busolt (88/89).
} 
Para nuestro filósofo, el genio se expresa por medio de sus productos, que son originales. Pero no es el caso que todos los originales sean dignos de ser imitados. Los productos de genio son originales y, además, dignos de ser imitados. Esto es crucial para Kant porque no es el caso que cualquiera que simplemente quiera no seguir ninguna regla haya de ser considerado genio. Kant no quiere dejar abierta la puerta para que cualquier tipo de irreverencia sea autorizada. El genio se libera de las reglas en la medida en que sus productos se conviertan en regla. El genio no es extravagante. Crea originales dignos de ser imitados. Si bien la idea general es la misma, en el transcurso de los años ochenta, empero, el ejemplo representativo de la noción de genio cambia: no es Shakespeare, sino Miguel Ángel y Milton.

Durante esta década, además, Kant es elogioso de Gerard en sus cursos, y recupera una noción de imaginación productiva, que movilizada por el espíritu vivificante se constituye como el rasgo principal del genio. En las lecciones inmediatamente anteriores a la publicación de la Crítica del Juicio, el modo como define al genio recupera ideas de las lecciones de los '70, más específicamente del libro que Kant usaba para enseñar la disciplina enciclopedia filosófica. El genio es una imaginación productiva sobresaliente, independiente, magistral, que sin embargo debe ser ordenada por el entendimiento, puesto que de lo contrario sus productos serían caóticos. El principio que la vivifica es el espíritu.

\section{La Crítica del Juicio}

Después de dar clases en varias cátedras durante tres décadas y de examinar los límites y el alcance de la razón pura en sus usos teórico y práctico, Kant publicó la Crítica del Juicio en 1790. En este texto desarrolló varios temas. El hilo conductor que los reúne es el examen de los elementos a priori de los juicios en los que el universal no está dado, es decir los juicios reflexionantes. El gusto parece haber sido la clave para que Kant identificara la necesidad de examinar si estos juicios reflexionantes requerían principios a priori. Para construir sus argumentos, Kant recuperó numerosos conceptos que había trabajado y discutido en sus clases. ${ }^{38}$

En rigor, la presentación de la doctrina del genio que encontramos aquí tiene algunas diferencias con respecto a la presentación de los cursos. Desde luego, la descripción de estas diferencias requiere un estudio muy minucioso. El genio se explica en el contexto del tratamiento del arte, que se encuentra inmediatamente después de la deducción del principio a priori de los juicios de gusto. En el tratamiento del arte podemos identificar tres momentos: 1) la explicación de la noción de arte, 2) la doctrina del genio, 3) el tratamiento de los productos del genio, es decir, la doctrina de las bellas artes. Vamos a concentrarnos en el segundo momento, pero para ello conviene tener presentes algunos aspectos del primero.

Cuando pensamos qué es el arte, podemos diferenciarlo de otros ámbitos de la experiencia. Por ejemplo, el arte no es naturaleza. Cuando un animal realiza una actividad para satisfacer sus necesidades, no pensamos que eso es arte, sino que establecemos una relación diferente entre el agente y el producto. En particular, consideramos que el agente

\footnotetext{
${ }^{38}$ Quizás la investigación más rigurosa de este proceso sea el libro de SÁNCHEZ RODRÍGUEZ (Sentimiento y reflexión en la filosofía de Kant. Estudio histórico sobre el problema estético), en el que examina la génesis de la KU en las lecciones de antropología.
} 
que produce la obra de arte tiene una intención. La obra de arte no se crea por instinto. Pero incluso entre las actividades mediadas por intenciones, consideramos que el arte es diverso de otras actividades: lo distinguimos de la ciencia y de la creación de artefactos en general. El artista tiene una intención pero no dispone de un concepto determinado de lo que la cosa que produce deba ser, ni dispone de reglas que le indiquen cómo producirla. Cuando juzgamos que algo es arte juzgamos que es el producto de intenciones, es decir que involucra una voluntad pero también consideramos que esa producción no está determinada por el concepto de un objeto, ni por instrucciones operativas ${ }^{39}$.

Una pregunta importante que debemos hacernos es: ¿ ¿es esta representación del arte compatible con la noción de belleza que se funda en el juicio reflexionante cuyo principio a prori ha sido deducido previamente, en la Crítica del Juicio? Si la respuesta a esta pregunta fuera que NO, la Crítica del Juicio tendría algunas dificultades. El primer problema que yo veo es que en este caso Kant habría encontrado un principio a priori que no vale para todos los juicios de gusto, sino sólo para los juicios de gusto sobre los objetos naturales. ¿En qué consisten en principio los juicios de gusto? ¿Qué decimos cuando decimos que un objeto es bello? Cuando digo "esto es bello", expreso que con ocasión de su representación en este momento experimento un sentimiento peculiar de placer y, además, afirmo que espero que ese mismo sentimiento de placer sea sentido por todos los otros seres que tienen mis facultades de conocimiento. ${ }^{40}$ Ahora bien, si la noción del arte no fuera compatible con las condiciones de los juicios de belleza, habría ciertos juicios de belleza para los que no valdría todo lo que sostiene Kant en la Crítica del Juicio, a saber, los juicios sobre obras de arte. Esto podría ser un poco escandaloso para las pretensiones ilustradas pero no sé si sería una objeción grave para el sistema filosófico de Kant. Ahora, hay un segundo problema más acuciante. Si el principio a priori de lo bello valiera sólo para el enjuiciamiento de la naturaleza, entonces el juicio de gusto involucraría una condición en la representación juzgada como bella. Una determinación conceptual, a saber la que dice "esto es un objeto natural", condicionaría el juicio de gusto. Y esto es algo no deseado.

Ahora bien, ¿por qué uno se preguntaría por la posibilidad de los juicios de gusto sobre artefactos? Hay numerosos pasajes en los que el mismo Kant señala dificultades al respecto. El ejemplo más claro es la nota al pie que se encuentra en la determinación de lo bello según el tercer momento. ${ }^{41}$ Es precisamente el tercer momento de la analítica de lo bello, el momento de la relación, el momento en el que entra en juego la noción de una conformidad a fin sin la representación de un fin, el que resulta problemático a la hora de pensar si y cómo es posible el juicio de belleza sobre artefactos.

Además Kant señala el problema de la noción de “arte bello". Esta noción refiere una representación que en cuanto que es artística es ella misma conforme a fin, en otras palabras: uno es consciente de que es arte y no naturaleza. Pero al mismo tiempo, en cuanto la juzgamos bella tenemos que ser capaces de sentir un placer que no se funde en conceptos. Kant menciona este conflicto en el siguiente pasaje de la Crítica del Juicio: "Pues todo arte presupone reglas a través de cuya fundamentación ante todo un producto, si es que debe denominarse artístico, se representa como posible. Pero el concepto del arte bello no permite

\footnotetext{
${ }^{39}$ Cf. KU \$43, AA 5:303s.

${ }^{40}$ Cf. KU $\$ 37$, AA 5: 289s.

${ }^{41}$ Estudié esta nota en MARTÍNEZ, L. "Una función del genio en la deducción del principio a priori de los juicios de gusto".
} 
que el juicio sobre la belleza de su producto sea derivado de alguna regla que tenga un concepto como fundamento de determinación". ${ }^{42}$

Aunque el producto sea intencional, tenemos que poder representarlo como si no lo fuera. Kant explica que la naturaleza es bella cuando parece arte. Nos representamos eso ahi como si estuviera dispuesto para satisfacernos, para ser conforme al libre juego de nuestras facultades. Y el arte sólo puede ser llamado bello cuando parece naturaleza. Nos representamos ese producto humano como si ningún concepto hubiera dirigido su producción, como si ninguna deliberación hubiera condicionado su producción. Es un producto del arte. El arte siempre tiene un propósito. Pero lo juzgamos como bello. Es decir, ese producto suscita un placer que juzgamos comunicable. La conformidad a fin no debe parecer intencional. Entonces, el objeto no debe mostrar huella alguna de que hay reglas que condicionan el ánimo del artista. Por lo tanto, el arte es bello cuando parece naturaleza. ${ }^{43}$ El problema, entonces, consiste en explicar cómo es posible que juzguemos que objetos artísticos son bellos- como, por lo demás, lo hacemos, de hecho. La manera que tenemos de representarnos el arte bello es precisamente ésta: considerar el arte como producto de genio. Genio es un don natural, es la naturaleza siendo prodigiosa en el hombre. No suponemos una regla humana, es decir conceptual, en el origen del arte, sino una regla puesta directamente por la naturaleza. Y es de tal índole que el genio ni siquiera puede explicar en qué consiste, ni cómo obtenerla. El genio es original, pues el origen de su obra es algo que la naturaleza pone en él y sólo en él. Para que sea concebible el enjuiciamiento estético del arte, es necesario suponer este tipo de representación de su origen: un genio que crea según una regla involuntaria, sin copiar nada más. Si copiara, entonces su obra tendría una regla intelectual y no sería bella: el criterio de su valoración sería la adecuación al modelo.

Desde luego, como la regla es un don natural, no puede enseñarse. Además de enfatizar en la originalidad del genio y en su origen natural, Kant retoma en la Crítica del Juicio un rasgo que ha explicado en sus cursos: el genio no se forja, no se aprende. Tampoco imita. Aprender es imitar, la aptitud para el aprendizaje no es genio. Por esta razón, no hay genio en la ciencia. Pero inventar tampoco es ser genio. En este punto, el Kant crítico se distancia de Gerard, para quien la capacidad del genio consiste en inventar. Para Kant se puede aprender a inventar, se puede desarrollar esta capacidad. El genio, en cambio, es algo dado por la naturaleza al hombre que nace y muere con él.

Ahora bien, es posible inquirir acerca de esto que es dado por la naturaleza al genio. Kant señala que la naturaleza da la regla para el arte ${ }^{44}$. Esa regla no puede formularse para servir como precepto. En caso contrario, el juicio de belleza podría determinarse por conceptos. La regla se puede conocer a posteriori, por abstracción, a partir del producto. Por medio de ella, los otros pueden testear su propio talento. Además, el genio sirve como patrón (Muster), como modelo. De esta manera, el genio se presenta como un modelo y su producto hace accesible una regla que sirve de prueba para el talento de los demás. Las ideas del artista suscitan ideas semejantes en sus discípulos, si éstos tienen proporciones semejantes de las fuerzas anímicas. ${ }^{45}$ Me parece que en esta indicación se encuentra una clave para comprender en qué consiste el don natural del genio.

\footnotetext{
$42 \mathrm{KU}$ \46, AA 5: 307.

${ }^{43} \mathrm{KU}$ \45, AA 5: 306s.

${ }^{44} \mathrm{KU}$, \46, AA 5: 307.

${ }^{45} \mathrm{KU}$, \46, AA 5: 308.
} 
Aunque ese don no pueda ser explicado ni enseñado, no debe pensarse en términos sobrenaturales. En rigor, esta noción de genio corresponde a algo que ya encontramos en Baumgarten, en Meier, en los libros que Kant leía para preparar sus clases y que explicaba en sus cursos: naturalmente, nuestras facultades del ánimo se encuentran en ciertas proporciones. ${ }^{46}$ Es en virtud de esas proporciones que tenemos talentos. Los talentos que tenemos son proporciones específicas en el desarrollo de alguna u otra de nuestras capacidades. Algunos aspectos de ellas pueden ser cultivados, pero no todos. El genio es una proporción que no puede ser cultivada.

Hay, sin embargo, para Kant una parte mecánica en la producción de arte. Esta parte sí puede enseñarse en las escuelas. En el texto crítico Kant recupera un espacio para las escuelas de arte. El genio, como talento original, es la parte esencial de la producción de arte bello, pero no la única. El genio proporciona el sustrato (Stoff) del producto. La forma de este producto, empero, requiere un talento cultivado. ${ }^{47}$ En este artículo nos interesa el aspecto de la producción de la obra que no se puede aprender, la parte que supone genio. Y todavía no somos capaces de especificar demasiado esta noción. Sabemos que es un don natural y que proporciona el sustrato de la obra.

Kant especifica más esta noción, a partir de una exégesis de la representación habitual de las bellas artes. ¿Qué es lo que percibimos cuando identificamos el arte bello? De los productos del arte de los que se espera que se muestren (sich zeigen) como bellos se dice que tienen o no tienen espíritu (Geist). Ahora bien, esta noción de espíritu, mientras no sea explicada, no arroja luz al problema. ¿Qué significa esto de tener espíritu? ¿Qué es el espíritu? Kant propone una definición. El término puede resultar polisémico. Pero en su significado estético, el espíritu es un "principio vivificante en el ánimo". ${ }^{48}$ Este principio nos permite exhibir ideas estéticas.

Las ideas estéticas son representaciones de la imaginación que no se pueden exponer. La exposición de una representación consiste en la exhibición clara y distinta de (algunos de) sus elementos. La razón por la que no se puede exponer ideas estéticas es que no hay conceptos que les correspondan. Estas representaciones de la imaginación no pueden ponerse en correspondencia con conceptos. No hay conceptos que sean adecuados a ellas. Por este motivo, las ideas estéticas son representaciones que tienen su origen en la imaginación y son inexponibles. Sin embargo, es posible exhibirlas. En particular, el espíritu es la capacidad de exhibir ideas estéticas. ${ }^{49}$ La noción misma de estas ideas tiene una doble determinación. Por una parte, ellas son un producto de la imaginación. Por esto Kant las denomina "estéticas". Se distinguen de las representaciones lógicas, que se originan en nuestras facultades para pensar. Sin embargo, son ideas. En este sentido, hay un vínculo en el nombre entre ellas y los conceptos que produce la razón en su uso puro, i.e. las ideas de la

\footnotetext{
${ }^{46}$ Cf. SÁNCHEZ RODRÍGUEZ, Sentimiento y reflexión en la filosofía de Kant. Estudio histórico sobre el problema estético, 168.

47 Jovanović (“A New Look at Kant's Genius: a Proposal of a Multi- componential Account”) encuentra en este punto una suerte de conflicto. Para la autora, el hecho de que el genio proporcione el sustrato de la obra, y que por consiguiente la forma de ésta corresponda al aspecto del arte que puede ser aprendido en las escuelas, es incompatible con la doctrina de las ideas estéticas (que desarrollaremos en este artículo, más adelante). Por el contrario, yo encuentro consistentes entre sí estos dos momentos del texto kantiano. Las ideas estéticas son parte del sustrato de la obra, son su contenido, son lo que ellas expresan. La forma de esta expresión es un producto de las técnicas, en las que el artista debe entrenarse.

${ }^{48} \mathrm{KU}$, 『 49 , AA 5: 313.

${ }^{49} \mathrm{KU}, \S 49, \mathrm{AA} 5: 314$
}

: : : Cadernos de Ética e Filosofia Política | Número 38 (1) | $1^{\circ}$ semestre de 2021 | Página 94 : : 
razón. La diferencia entre ambos tipos de ideas está dada por el origen: la imaginación y la razón pura, respectivamente. La similitud, el motivo por el que Kant las denomina del mismo modo, está dada por la tendencia de ambas clases de ideas de remitir a algo más allá de los límites de la experiencia.

Las ideas estéticas tienen su origen en una facultad común: la imaginación. Las ideas estéticas son el producto de un talento, una imaginación bien desarrollada. Pero su presentación requiere algo más: requiere espíritu. El genio es una disposición tal de las facultades, que consigue encontrar ideas para un concepto dado y expresarlas de tal manera que comuniquen a otros una disposición subjetiva del ánimo. La capacidad de expresión de ideas estéticas es el espíritu.

Así, el genio tiene un talento especialmente desarrollado. Se trata de la imaginación, que le proporciona ideas estéticas. Pero además tiene la capacidad de expresar estas representaciones que de otro modo serían inefables; es decir, el genio tiene espíritu. Kant concluye que "Puede explicarse el genio mediante la facultad de las ideas estéticas, con lo cual se indica al mismo tiempo el fundamento por el que en los productos del genio la naturaleza (del sujeto), y no un fin meditado, da la regla al arte (para la producción de lo bello)." ${ }^{50}$ De esta manera, la doctrina del genio resuelve el problema de la posibilidad del arte bello.

Adicionalmente, conviene tener presente que esta resolución tiene una virtud significativa, relevante para el razonamiento sistemático del texto en el que se inscribe. La doctrina del genio, en pocas palabras, hace que podamos comprender la belleza natural y la belleza artística de la misma manera, es decir que sean concebibles los juicios de gusto no condicionados por el origen de la representación juzgada. Este corolario se señala en el siguiente pasaje: "En general, puede llamarse belleza (sea de la naturaleza o del arte) a la expresión (Ausdruck) de ideas estéticas." ${ }^{51}$

Hemos visto que en las décadas previas a la publicación de la Crítica del Juicio Kant había realizado un esfuerzo, un enorme trabajo conceptual para especificar la noción del genio. En el texto de la Crítica, esta noción se presenta como una noción precisamente determinada, con una función sistemática clave. El genio de la Crítica del Juicio tiene una imaginación rica que produce ideas estéticas, pero tiene sobre todo el espíritu, que es la capacidad de expresarlas. Pienso que esta noción del genio poco o nada tiene que ver con la producción de conocimiento o las invenciones ingeniosas. Pero esto no es un problema, no constituye una limitación conceptual, pues la antropología de Kant cuenta con otros conceptos para explicar los otros talentos del hombre.

\section{Recapitulación}

En la introducción de este artículo se han revisado las principales ideas desarrolladas en Wenzel (2001). Luego, en el cuerpo del texto se han examinado dos clases de fuentes. En primer término, se han examinado las fuentes disponibles correspondientes al período previo a la publicación de la Crítica del Juicio. En segundo lugar, hemos revisado las ideas desarrolladas

\footnotetext{
${ }^{50} \mathrm{KU}, \S 57$, Obs. 1, AA 5: 344.

${ }^{51} \mathrm{KU}, \int 51$, AA 5:320
}

: : : Cadernos de Ética e Filosofia Política | Número 38 (1) | 1º semestre de 2021 | Página 95 : : : 
en este texto publicado. En lo que queda, recorreremos algunas tesis de Wenzel que contrastan con la exégesis desarrollada.

En primer lugar, advertimos que para Wenzel con la Crítica del Juicio se produce una ruptura respecto de las posiciones kantianas antecedentes en relación con la necesidad de genio para el conocimiento matemático. Nuestro recorrido por las fuentes muestra que ya a comienzos de los años '70, dos décadas antes de la publicación de la Crítica del Juicio, Kant consideraba que no hay genio en matemática. ${ }^{52}$ En el período precrítico y hasta comienzos de la década de 1780, empero, el filósofo contempló la posibilidad de reconocer que hay genio en el ámbito del conocimiento. Esto se modifica, de acuerdo con los testimonios disponibles, a comienzos de la década de 1780. En este momento, Kant afirma de manera explícita que la esfera de incumbencia del genio es la producción artística. En este momento, Kant ha leído a Gerard y ha introducido algunas novedades en sus cursos. Por un lado, ha comenzado a especificar las diferentes facultades que constituyen el genio. Por el otro lado, ha resaltado entre ellas el espíritu, que constituye una suerte de vivificación de todas.

Además, Wenzel vincula el cambio señalado con dos aspectos de la doctrina del genio en el texto crítico. En primer lugar, el genio se vincula con la producción de la imaginación. El foco en esta facultad es, sí, un rasgo característico del concepto de genio de Gerard. Kant pone, también, la imaginación en un lugar central de la doctrina. En la Crítica empero este lugar, en el que la imaginación es el origen de las ideas estéticas, no es solitario. Junto a la imaginación, el filósofo identifica otras capacidades del genio ${ }^{53}$. Entre ellas se ha destacado aquí una disposición vivificante por la que el genio es apto para expresar las ideas estéticas de la imaginación. Es decir, junto con esta facultad reconocemos el espíritu. El segundo cambio que Wenzel identifica en el criticismo está dado por la diferenciación entre el genio y los talentos. Ciertamente, desde los'70 y durante el criticismo Kant es enfático sobre el carácter original del genio, que no puede ser aprendido. En esto, el genio se diferencia de otras habilidades y talentos. No obstante ello, en la Crítica del Juicio Kant dice explícitamente que el genio tiene un talento ${ }^{54}$, que es un don natural.

A partir de la lectura de las fuentes que describo en este artículo, pienso que podemos concluir que se produce una necesaria especificación del concepto de genio en la Crítica del Juicio. Esta especificación está vinculada con la función que tiene ese concepto en el contexto

\footnotetext{
${ }^{52}$ Se pueden identificar empero algunos pasajes aislados, por ejemplo en V-Anth/ Fried, en los cuales Kant menciona la posibilidad de algún tipo de genio matemático. Esto se presenta como un requerimiento excepcional y no exhibe la consideración general del filósofo, que ha sido desarrollada en la sección correspondiente de este artículo.

${ }^{53}$ En este trabajo me demoré solamente en la imaginación y el espíritu. No son los únicos elementos constitutivos del genio, ni los más problemáticos. Un tema de debate es, también, la relación entre genio y gusto. Esta relación era ya discutida en la época de Kant y ocupó la pluma de pensadores célebres como Gerard y Herder. Alexander Pope ha descrito al genio como una dimensión irreductible entre el gusto y el aprendizaje (An Essay on Criticism, 48s.) Para Gerard, el genio y el gusto surgen de la imaginación y tienen una relación inestable y variable (An Essay on Taste with three dissertations on the same subject by Voltaire, D'Alembert, Montesquieu, 177). Posteriormente, Herder estableció la prioridad del gusto. Éste es considerado por Herder como un presupuesto para el genio. El gusto se define como un conjunto de facultades, en tanto que el genio se considera como una suerte de ordenamiento de ellas. ("Ursachen des gesunknen Geschmacks bei den verschidnen Völkern, da er geblühet”, 158). Actualmente, también los comentadores intentan explicar la relación entre ambos en la Crítica del Juicio. Jovanović ("A New Look at Kant's Genius: a Proposal of a Multi- componential Account") propone una respuesta que a mí me resulta insatisfactoria. Para ella, hay un conflicto entre genio y gusto en el texto de Kant que puede reformularse en términos de discutir si el gusto es exterior o interno al genio.

${ }_{54} \mathrm{KU}$, \$46, AA 5: 307.
} 
general de la argumentación desarrollada allí. El concepto de genio, que incluye la producción y la expresión de las ideas estéticas, facilita un abordaje de la belleza artística que resulta compatible con la caracterización del Juicio reflexionante estético.

\section{Referencias bibliográficas}

BAUMgarTEN, A. G. Metaphysik. Ins Deutsche übersetzt von Georg Friedrich Meier. Jena: Dietrich Scheglmann Reprints, 2004.

BECKENKAMP, J. "Kant und Gerard über Einbildungskraft”. In: Dörflinger, B.; La Rocca, C.; Louden, R.; de Azevedo Marques, U. R., Kant's Lectures / Kants Vorlesungen. Berlin: Walter de Gruyter, 2015.

“Kant e Gerard sobre imaginação”. In: Studia Kantiana, 20, 117-127, 2016.

DESMOND, W. "Kant and the Terror of Genius: Between Enlightenment and Romanticism". In: Kants Ästhetik - Kant's Aesthetics · L'esthétique de Kant. Berlin, Boston: De Gruyter, 594-614, 2013.

FEDER, J. G. Grundriß der Philosophischen Wissenschaften nebst der nöthigen Geschichte. Coburg : Findeisen, 1769.

FRICKE, C. “Genius”. In: Willaschek, M., Stolzenberg, J., Mohr, G., Bacin, S., Kant-Lexikon. Berlin, Boston: De Gruyter, 756-758, 2015.

GERARD, A. An Essay on Taste with three dissertations on the same subject by Voltaire, D'Alembert, Montesquieu. London: Millar, Kincaid, and Bell, 1759.

An Essay on Genius. München: Wilhelm Fink Verlag, 1966.

GIORDANETTI, P. "Kant e Gerard. Nota sulle fonti storiche della teoria kantiana del 'genio"”. In: Rivista Di Storia Della Filosofia 46 (4), 661-699, 1991.

"Das Verhältnis von genius, Künstler und Wissenschaftler in der Kantischen Philosophie”. In: Kant-Studien, 86(4), 406-430, 1995.

HERDER, J. G. “Shakespeare”. In: Von Deutscher Art und Kunst. Hamburg, 73-118, 1773.

"Ursachen des gesunknen Geschmacks bei den verschidnen Völkern, da er geblühet”. In: Herders Werke in fünf Bänden, drittes Band. Berlin und Weimar: Aufbau Verlag, 1964.

JOVANOVIĆ, I. “A New Look at Kant's Genius: a Proposal of a Multi- componential Account”. Con-Textos Kantianos. International Journal of Philosophy, 1(12), 248-269, 2020. 
KANT, I. Gesammelte Schriften. Hrsg.: Bd. 1-22 Preussische Akademie der Wissenschaften, Bd. 23 Deutsche Akademie der Wissenschaften zu Berlin, ab Bd. 24 Akademie der Wissenschaften zu Göttingen. Berlin 1900 ss.

KLUKOFF, P. J. “Alexander Gerard. An Essay on Genius (1774)". In: Studies in Scottish Literature, 5 (3), 201-202. Edited by Bernhard Fabian (Theorie und Geschichte der Literatur und der Schönen Künste, vol. III). München. Wilhelm Fink Verlag. 1966. vii + 459 pp. DM.52”, 1967.

MARTÍNEZ, L. "El desarrollo del genio artístico". In: Con-Textos Kantianos, 11, 176-190, 2020.

"Die Lehre vom Genie in Kants Vorlesungen über Philosophische Enzyklopädie". In: Weibel, V. y Ruffin, M. (ed.), Nature and Freedom/ Natur und Freibeit/ Nature et Liberté, Berlín: De Gruyter, 901-908, 2019.

- "Una función del genio en la deducción del principio a priori de los juicios de gusto”. In: Órdenes, P. y Alegría, D. (ed.), Kant y el Criticismo. Presente, pasado, ¿futuro?, Porto Alegre: Editora Fi, 2015.

ORTLAND, E. "Genius". In: Barck, K. et al, Ästhetische Grundbegriffe, Band 2. Stuttgart, Weimar: Metzler Verlag, 2001.

POPE, A. An Essay on Criticism. London, 1711.

SÁNCHEZ RODRÍGUEZ, M. Sentimiento y reflexión en la filosofía de Kant. Estudio bistórico sobre el problema estético. Hildesheim, Zürich, New York: Georg Olms Verlag, 2010.

TONELLI, G. "Kant's Early Theory of Genius (1770-1779): Part I”. In: Journal of the History of Philosophy, 2, 1966.

. "Kant's Early Theory of Genius (1770-1779): Part I". In: Journal of the History of Philosophy, 4(3), 209-224, 1966.

WENZEL, C. H. "Beauty, Genius, and Mathematics: Why Did Kant Change His Mind?". In: History of Philosophy Quarterly, 18(4), 415-432, 2001.

YOUNG, E. Conjectures on Original Composition. Manchester: The University Press, London, New York, etc.: Longmans, Green \& Co, 1918. 